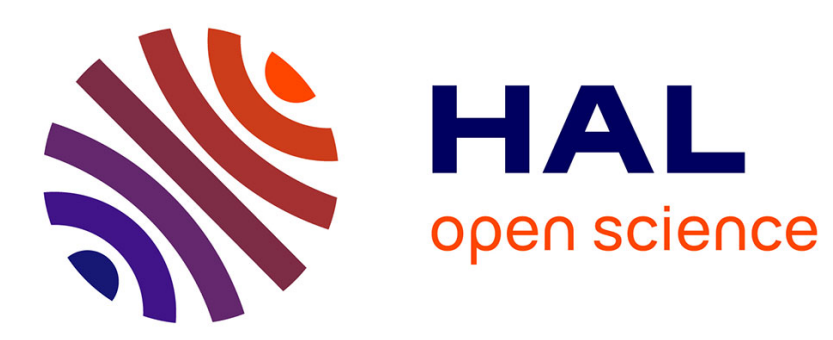

\title{
MiPNO, a new chiral nitrone for enantioselective amino acid synthesis: the cycloaddition approach.
}

Maryse Thiverny, Christian Philouze, Pierre Yves Chavant, Véronique Blandin

\section{To cite this version:}

Maryse Thiverny, Christian Philouze, Pierre Yves Chavant, Véronique Blandin. MiPNO, a new chiral nitrone for enantioselective amino acid synthesis: the cycloaddition approach.. Organic \& Biomolecular Chemistry, 2009, 2010 (8), pp.864-872. 10.1039/b918612c . hal-00453930

\section{HAL Id: hal-00453930 \\ https://hal.science/hal-00453930}

Submitted on 11 Sep 2012

HAL is a multi-disciplinary open access archive for the deposit and dissemination of scientific research documents, whether they are published or not. The documents may come from teaching and research institutions in France or abroad, or from public or private research centers.
L'archive ouverte pluridisciplinaire HAL, est destinée au dépôt et à la diffusion de documents scientifiques de niveau recherche, publiés ou non, émanant des établissements d'enseignement et de recherche français ou étrangers, des laboratoires publics ou privés. 


\title{
Peer-reviewed manuscript of the article published in
}

\section{Organic \& Biomolecular Chemistry, 2010, 8, 864-872}

Reproduced by permission of The Royal Society of Chemistry

http://www.rsc.org/publishing/journals/OB/article.asp?doi=b918612c

DOI: $10.1039 / b 918612 c$

\section{MiPNO, a new chiral cyclic nitrone for enantioselective amino acid synthesis: the cycloaddition approach.}

\author{
Maryse Thiverny, Christian Philouze, Pierre Yves Chavant and Véronique Blandin*
}

The resolution of chiral nitrones via derivatization of hydroxylamines was applied to MiPNO, a new, stable, easily prepared chiral cyclic nitrone. The application of MiPNO in totally regio- and diastereo- selective 1,3-dipolar cycloaddition reactions provides an expeditious enantioselective access to unusual $\gamma$-hydroxy $\alpha$-aminoacids.

\section{Introduction}

The regio- and diastereo- selective 1,3-dipolar cycloaddition reaction of chiral nitrones with dipolarophiles is a powerful tool to obtain chiral nitrogenated compounds. ${ }^{1}$ In particular, when $\alpha$-alkoxycarbonyl or $\alpha$-alkylaminocarbonyl nitrones are at stake, the cycloaddition reaction with alkenes followed by cleavage of the $\mathrm{N}-\mathrm{O}$ bond leads to side chain-functionalized chiral $\alpha$-amino acid derivatives. ${ }^{2}$ In order to gain a high stereoselectivity in the cycloaddition reaction, overcoming the problem of $E / Z$ isomerization around the nitrone double bond is important. ${ }^{3}$ Small-ring endocyclic chiral nitrones are thus good candidates and their more rigid cyclic skeleton compared to acyclic nitrones is another asset to obtain high stereoselectivity. Several five- ${ }^{4}$ and six- ${ }^{5}$ membered chiral cyclic nitrones of this type have been previously designed. ${ }^{6}$

In parallel to our work in the field of $N$-hydroxy peptides synthesis and application, ${ }^{7}$ we sought an access to $N$-hydroxy amino acids via the addition of functionalized organometallic reagents ${ }^{8}$ on to properly designed enantiopure nitrones. To this end, we designed a series of chiral nitrones ${ }^{4 \mathrm{~d}, \mathrm{e}}$ obtained by oxydation of imidazolidinones and based on the self-regeneration of stereocenters principle. ${ }^{9}$ In our continuous effort to provide storable reagents and intermediates using inexpensive starting materials and scalable syntheses, ${ }^{10}$ we developed the new chiral nitrone 1 (MiPNO), that turned out to feature an excellent thermal stability. This made 1 a good candidate for 1,3-dipolar cycloaddition reactions that usually require prolonged heating. We report herein the synthesis of $\mathbf{1}$ and its evaluation as a successful substrate for diastereoselective 1,3-dipolar cycloaddition reactions.

\section{Results and discussion}

Nitrone 1 was conveniently prepared in three steps according to Scheme 1, starting from glycine ethyl ester hydrochloride. Condensation of amino amide $\mathbf{2}$ with 3-methyl-butanone led exclusively to the desired cyclic product $\mathbf{3}$. Oxidation of $\mathbf{3}$ was performed using urea-hydrogen peroxide (UHP) complex in the presence of inexpensive sodium tungstate as the catalyst. ${ }^{11}$ The whole process was routinely carried out on a 200 mmoles scale. After recrystallization the yield of 1, over three steps, was typically 54\%. Nitrone 1 could be stored at room temperature for months without noticeable degradation. 

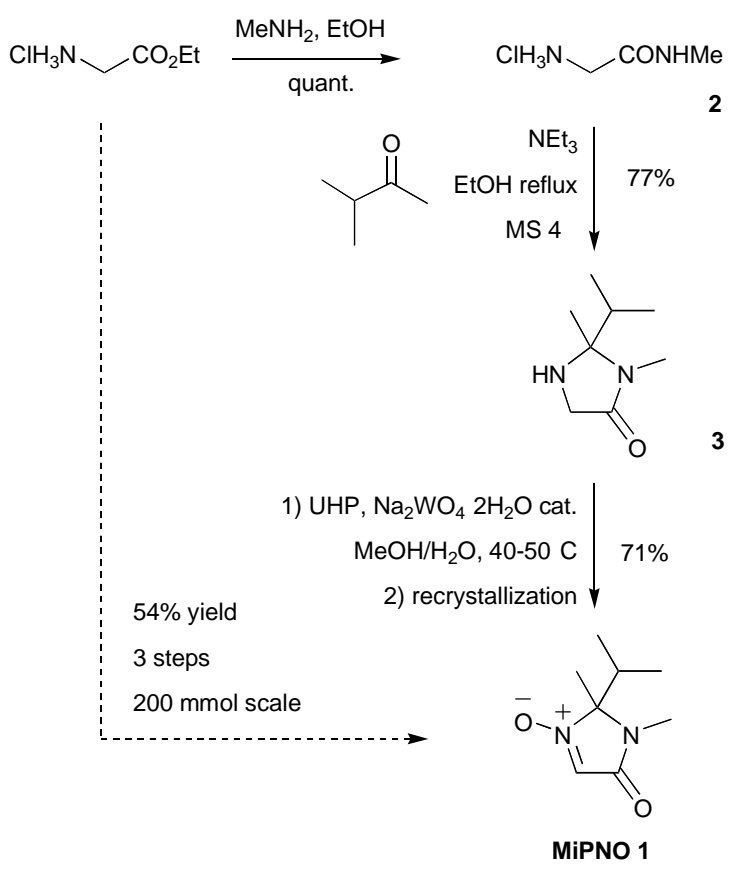

Scheme 1. Preparation of MiPNO 1.

X-ray analysis of $\mathbf{1}^{12}$ showed a planar ring with one side hindered by the isopropyl group. In order to evaluate its level of diastereoinduction, nitrone 1 was engaged in 1,3-dipolar cycloaddition reactions with a wide range of alkenes (Table 1), either in neat conditions or using toluene as the solvent. ${ }^{13}$ The reaction was highly regio- and diastereo- selective, the nitrone reacting from the less hindered side in an exo mode to give cycloadducts 5a-h. ${ }^{14}$ The NMR and LC/MS analysis of the crude products indicated an isomeric purity of more than $98 \%$ in all cases except in case of the acetal 5d (table 1, entry 4). Nevertheless, after chromatographic purification all compounds were isolated as pure cycloadducts in excellent yield.

Alkyne dipolarophiles are seldom reported in the reaction with nitrones ${ }^{1 \mathrm{a}}$ and the cycloadducts are prone to rearrangements. ${ }^{5 \mathrm{c}}$ In our hands, no reaction occured between nitrone $\mathbf{1}$ and propargylic alcohol or protected derivatives thereof. However, phenylacetylene reacted smoothly to give the expected cycloadduct 7 in $73 \%$ isolated yield (entry 9). An open-chain enol-imine or keto-enamine by-product 7' derived from 7 through $\beta$-elimination ${ }^{15}$ was also isolated in $20 \%$ yield (Scheme 2).

Table 1. 1,3-dipolar cycloaddition of racemic nitrone $\mathbf{1}$ to alkenes $\mathbf{4 a - h}$ and alkyne $\mathbf{6}$.

\begin{tabular}{|c|c|c|c|}
\hline entry & $\begin{array}{c}\text { dipolarophile } \\
\mathbf{4} \text { or } \mathbf{6} \\
\end{array}$ & cycloadduct $^{\mathrm{a}}$ & yield $(\%)^{\mathrm{b}}$ \\
\hline 1 & $4 a$ & $5 a$ & $\begin{array}{c}87 \\
(94)^{c}\end{array}$ \\
\hline 2 & $4 b$ & $5 b$ & $\begin{array}{c}94 \\
(86)^{c}\end{array}$ \\
\hline 3 & $4 c$ & $5 c$ & 90 \\
\hline
\end{tabular}


4<smiles>C=COCCCC</smiles>

4d

5<smiles>C=CC(=O)OCC</smiles>

$4 e$

6<smiles>C/C=C/C(=O)OC</smiles>

7

8<smiles>C1=CCCCC1</smiles>

$4 \mathrm{~g}$

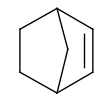

$4 h$

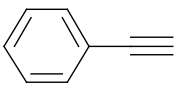

6<smiles>CCCCO[C@H]1C[C@@H]2C(=O)N(C)[C@](C)(C(C)C)N2O1</smiles>

$5 d$<smiles>CCOC[C@H]1C[C@H]2C(=O)N(C)[C@](C)(C(C)C)N2O1</smiles>

5 e<smiles>CO[C@@H]1[C@H]2C(=O)N(C)[C@](C)(C(C)C)N2O[C@@H]1C</smiles>

$5 f$<smiles>CC(C)[C@@]1(C)N(C)C(=O)[C@@H]2[C@H]3CCCC[C@H]3ON21</smiles>

$5 g$

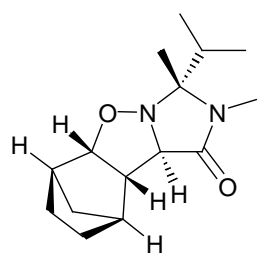

5 h<smiles>CC(C)[C@]1(C)N(C)C(=O)[C@H]2C=C(c3ccccc3)ON21</smiles>

7

${ }^{a}$ Unless otherwise stated, reactions were performed in a sealed vessel at $80{ }^{\circ} \mathrm{C}$ for $3-16 \mathrm{~h}$, using 10 equiv 4 or 6 without solvent. The main isomer, representing $\geq 98 \%$ of the cycloadducts in the crude product (LC/MS), is shown. ${ }^{b}$ Yield of the isolated main isomer. ${ }^{c}$ Starting from $(-)-(R)-1 .{ }^{d}$ Isomeric purity of the crude product $\geq 95 \%{ }^{e}{ }^{e}$ Toluene was used as a cosolvent. Reaction time: $48 \mathrm{~h}, 90 \%$ conversion. ${ }^{f} 1.2$ equiv alkene were used, in toluene. ${ }^{g} 2$ equiv alkyne were used, in toluene. The monocyclic isomer 7 ' resulting from $\beta$-elimination was isolated in $20 \%$ yield.

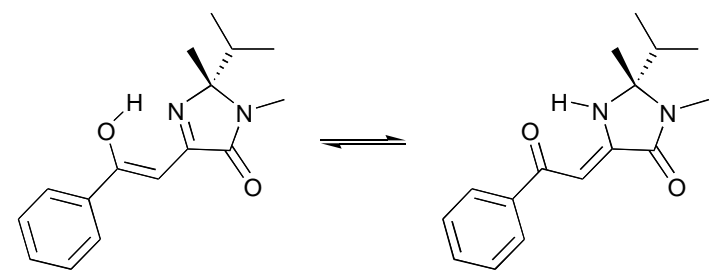

Scheme 2. Tautomeric structures of by-product 7'.

To elaborate the amino acid side chain further, reductive cleavage of the $\mathrm{N}-\mathrm{O}$ bond was investigated (Scheme 3 ). Hydrogenolysis of $\mathbf{5 e}$ and $\mathbf{5 f}$ under transfer conditions afforded amino alcohols $\mathbf{8 e}$ and $\mathbf{8 f}$ in good isolated yield. In the case of $\mathbf{5 f}$, a 9:1 mixture of diastereomers was obtained. ${ }^{16}$ For the styrene-derived cycloadduct $\mathbf{5 c}$, the reductive cleavage was performed 
using zinc in acetic acid, leading to amino alcohol 8c in excellent yield.

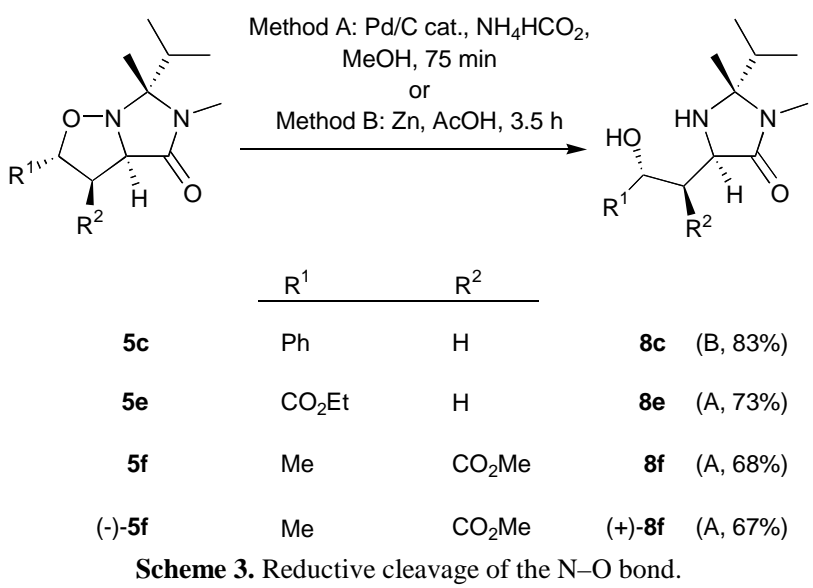

In order to obtain MiPNO 1 in enantiomerically pure form, we decided to use a strategy under development in our group, namely the resolution of secondary hydroxylamines via $O$-acylation with a chiral carboxylic acid. ${ }^{17}$ The nitrone 1 was thus converted into the $N$-hydroxy-imidazolidinone 9 by reduction with sodium borohydride (Scheme 4).

Interestingly, this reduction was clean when THF was the solvent, whereas the reduction in $\mathrm{MeOH}$ was accompanied by the formation in large amount $(30 \%)$ of the imine resulting from dehydration of 9. Moc-Phe-OH ${ }^{18}$ was chosen as the chiral derivatizing agent for its ability to provide chromatographically separable diastereomers. The 1:1 mixture of diastereomers $\mathbf{1 0}$ was obtained in high yield.

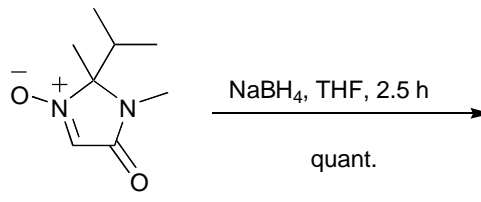

1

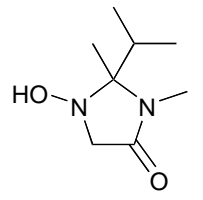

9

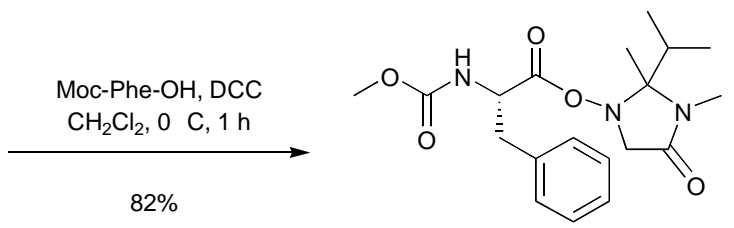

10

Scheme 4. Resolution of MiPNO: derivatization of hydroxylamine 9.

Unfortunately, the $O$-acylated hydroxylamine proved to be very sensitive to silica gel and partially decomposed into Moc-Phe$\mathrm{OH}$ and imine. Nevertheless, submitting the mixture of diastereomers to medium pressure liquid chromatography allowed the isolation of the first eluted diastereomer 10a as a solid in $32 \%$ yield. On the basis of the relative configuration obtained by X-ray analysis of recrystallized 10a (Figure 1$),{ }^{19}$ the stereochemistry of the imidazolidinone moiety is $(R)$. 


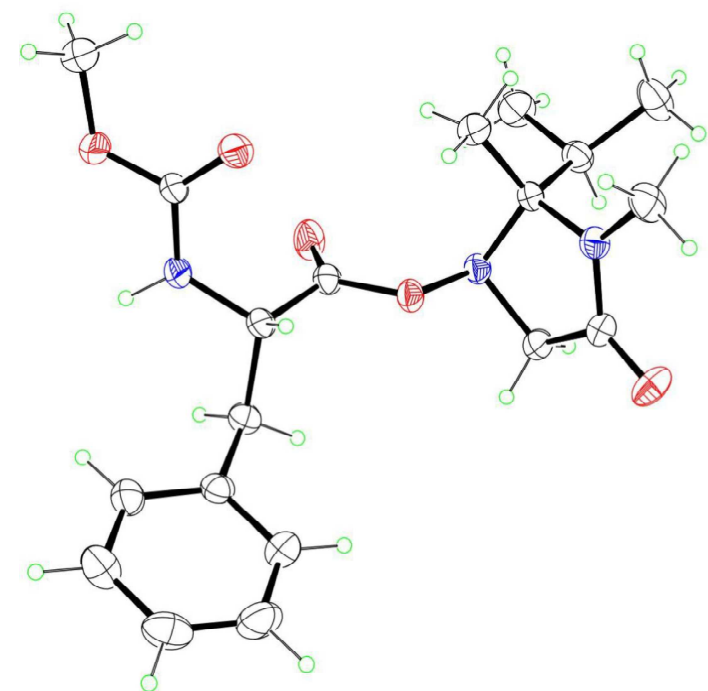

Figure 1. ORTEP drawing of 10a. The ellipsoids are plotted at the $25 \%$ probability level.

Treatment of 10a with basic aqueous hydrogen peroxide overnight allowed the one-pot liberation of the hydroxylamine and its oxidation into the nitrone (-)- $(R)-\mathbf{1}$. More efficiently, $\mathrm{MnO}_{2}$ may be added as soon as the first step is completed in order to accelerate the process (Scheme 5).

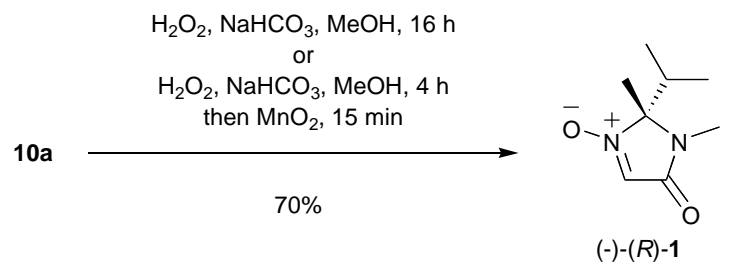

Scheme 5. Resolution of MiPNO: one-pot removal of resolving agent and oxidation.

The enantiomeric excess of (-)-1 was above $99 \%$ as determined by chiral HPLC analysis. The nitrone (-)-1 proved to be configurationally stable when purified by chromatography on silica gel $l^{4 \mathrm{c}}$ or when heated in toluene at $80{ }^{\circ} \mathrm{C}$ for $16 \mathrm{~h}$.

The 1,3-dipolar cycloaddition of enantiopure MiPNO (-)-1 with several alkenes gave results consistent with those obtained for the racemic series (Table 1, entries 1, 2, 6 and 7). The N-O bond of (-)-5f was cleaved using method A (Scheme 2) and (+)-8f was obtained in $67 \%$ yield. Next, the tricyclic compound (+)-5g was chosen to investigate the transformation of cycloadducts into conveniently $N$-protected amino acids (Scheme 6). We developed a microwave-assisted procedure where acidic hydrogenolytic conditions allow the one-pot cleavage of the $\mathrm{N}-\mathrm{O}$, amide, and $\mathrm{N}-\mathrm{C}-\mathrm{N}$ bonds. ${ }^{20}$ The crude $\alpha$-amino- $\gamma$-lactone ${ }^{21}$ was Boc-protected to give after chromatography (-)-11 as a single isomer in $42 \%$ yield from (+)-5g. This is to our knowledge the first preparation of 11 in enantiopure form.

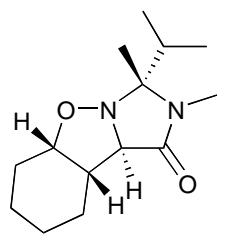

$5 \mathrm{~g}$

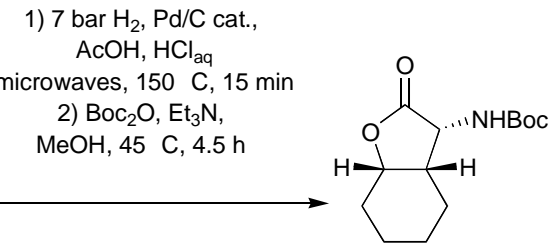

$(-)-11$

Scheme 6. One-pot conversion of cycloadduct $\mathbf{5 g}$ to $N$-protected $\alpha$-amino $\gamma$-lactone (-)-11.

\section{Conclusion}

The new nitrone MiPNO 1, easily prepared on large scale in three inexpensive steps and one recrystallization, has proven to exibit a high degree of facial differentiation, making it a promising new building block for the synthesis of $\alpha$-amino acids. The cycloaddition reaction with alkenes features excellent regio- and stereo- selectivity, affording a single enantiopure isomer in high yields. From there, one operation provides $\alpha$-amino $\gamma$-lactones, a masked form of $\gamma$-hydroxy $\alpha$-amino acids. We are currently studying improved resolutions of MiPNO, as well as its applications to other addition reactions. 


\section{Experimental}

\section{General}

THF was freshly distilled from sodium benzophenone ketyl. Styrene, cyclohexene, methyl crotonate and phenylacetylene were distilled before use, other purchased reagents were used without purification. Unless otherwise stated, the indicated reaction temperatures are external ones (oil bath). Reactions were monitored by thin layer chromatography (TLC) using commercial aluminium-backed silica gel plates (Merck, Kieselgel $60 \mathrm{~F}_{254}$ ). TLC spots were viewed under ultraviolet light and by heating the plate after treatment with an appropriate staining solution $\left(\mathrm{KMnO}_{4}\right.$, ninhydrine for amines, basic TTC (2,3,5-triphenyltetrazolium chloride) for hydroxylamines). Product purification by column chromatography were performed using Macherey Nagel Silica Gel 60M (230-400 mesh). Microwave irradiation experiments were conducted in a CEM Discover S-Class apparatus (single mode technology), equipped with a gas addition kit. Melting points were determined in capillary tubes with a Büchi B-540 apparatus. Optical rotations were measured on a Perkin Elmer 341 polarimeter, the corresponding concentration is given in $\mathrm{g} / 100 \mathrm{~cm}^{-3} .{ }^{1} \mathrm{H}$ NMR (400 MHz) and ${ }^{13} \mathrm{C}$ NMR $(100 \mathrm{MHz})$ spectra were recorded on a Varian 400MR spectrometer in $\mathrm{CDCl}_{3}\left(\delta_{\mathrm{H}} 7.26 \mathrm{ppm} ; \delta_{\mathrm{C}}\right.$ $77.2 \mathrm{ppm}$; standard for ${ }^{1} \mathrm{H}$ spectra: tetramethylsilane $\delta_{\mathrm{H}} 0.0 \mathrm{ppm}$ ). NOESY experiments were run on a Unity Plus $500 \mathrm{MHz}$ Varian. Multiplicities are reported as follows: $\mathrm{s}=$ singlet, $\mathrm{d}=$ doublet, $\mathrm{t}=$ triplet, $\mathrm{q}=$ quadruplet, quint $=$ quintuplet, hept $=$ heptuplet, $\mathrm{m}=$ multiplet, br = broad, coupling constants $J$ are reported in $\mathrm{Hz} .{ }^{1} \mathrm{H}$ and ${ }^{13} \mathrm{C}$ resonance assignments were performed using conventional 1D and 2D techniques (DEPT, COSY, HMQC and HMBC experiments). Relative stereochemistry was assigned using NOESY experiments. Infrared spectra (IR) were obtained either from a thin film or from a dispersion of the compound in a $\mathrm{KBr}$ pellet. IR spectra were recorded on a Nicolet Impact-400 FT-IR spectrometer and the data are reported as absorption maxima in $\mathrm{cm}^{-1}$. Mass spectra (LRSM) were recorded on an Esquire 300 Plus Bruker Daltonics spectrometer (ESI). High resolution mass spectra (HRMS) were recorded on a Thermoquest Orbitrap spectrometer at the LCOSB, UMR 7613, Université Pierre et Marie Curie, Paris, France. Experimental errors for HRMS data are estimated between 1 and 2 ppm.

\section{(rac)-2-Isopropyl-2,3-dimethyl-imidazolidin-4-one 3}

The aminoamide 2 was prepared according to ref. 22: in a 250-mL Erlenmeyer flask under magnetical stirring, methylamine (125 $\mathrm{mL}, 8 \mathrm{M}$ in ethanol, $1.0 \mathrm{~mol})$ was added to glycine ethyl ester hydrochloride $(27.92 \mathrm{~g}, 200 \mathrm{mmol})$ in ethanol $(80 \mathrm{~mL})$. After stirring for $16 \mathrm{~h}$ at room temperature, and concentration, the crude product was taken up in ethanol $(200 \mathrm{~mL})$ and the solvent and excess methylamine were removed under vacuum. This operation was repeated twice to give pure 2 (24.91 g, $200 \mathrm{mmol}$, quantitative yield) as an amorphous solid.

In a 500-mL flask fitted with a reflux condenser were introduced the aminoamide hydrochloride 2 (24.91 g, $200 \mathrm{mmol})$, ethanol $(200 \mathrm{~mL}), 3$-methyl-2-butanone $(42.8 \mathrm{~mL}, 400 \mathrm{mmol})$, triethylamine $(28.1 \mathrm{~mL}, 200 \mathrm{mmol})$ and activated $4 \AA$ molecular sieves (beads, $60 \mathrm{~g}$ ). The reaction mixture was heated for $16 \mathrm{~h}$ at reflux without stirring, then the sieves were filtered off and the solvent was evaporated under reduced pressure. The crude white slurry was taken up in ethyl acetate $(150 \mathrm{~mL})$, the precipitated triethylamine hydrochloride was filtered off and the solvent evaporated under reduced pressure to yield $24.15 \mathrm{~g}$ of crude material 3 (theoretical: $31.25 \mathrm{~g}$ ); $R_{f}=0.33$ (ethyl acetate/ethanol - 80:20); $v_{\max }(\mathrm{KBr}$ pellet): 3327, 2970, 2942, 2876, 1685, 1432,1404 and $1124 ; \delta_{\mathrm{H}}\left(400 \mathrm{MHz} ; \mathrm{CDCl}_{3} ; \mathrm{Me}_{4} \mathrm{Si}\right) 3.53(\mathrm{~d}, J 16.0,1 \mathrm{H}), 3.42(\mathrm{~d}, J 16.0,1 \mathrm{H}), 2.74(\mathrm{~s}, 3 \mathrm{H}), 1.91(\mathrm{qq}, J 6.4$ and $6.8,1 \mathrm{H}), 1.71(\mathrm{br}$ $\mathrm{s}, 1 \mathrm{H}), 1.34(\mathrm{~s}, 3 \mathrm{H}), 0.96(\mathrm{~d}, J 6.4,3 \mathrm{H}), 0.79(\mathrm{~d}, J 6.8,3 \mathrm{H}) ; \delta_{\mathrm{C}}\left(100 \mathrm{MHz}, \mathrm{CDCl}_{3}\right) \quad 172.7,82.0,49.4,34.7,25.2,24.2,16.6,16.2$ ppm; MS $\left(\mathrm{ES}^{+}\right): m / z 335\left(2 \mathrm{M}+\mathrm{Na}^{+}, 30 \%\right), 179\left(\mathrm{M}+\mathrm{Na}^{+}, 55\right), 157\left(\mathrm{M}+\mathrm{H}^{+}, 100\right) ; \mathrm{HRMS}: m / z .157 .13329(\mathrm{M}+\mathrm{H}) \mathrm{C}_{8} \mathrm{H}_{17} \mathrm{~N}_{2} \mathrm{O}$ requires 157.13354 .

\section{(rac)-2-Isopropyl-2,3-dimethyl-1-oxy-2,3-dihydro-imidazol-4-one 1}

In a 500-mL flask fitted with a reflux condenser the crude amine 3 (24.15 g, $154.58 \mathrm{mmol})$ was dissolved in methanol (200 $\mathrm{mL})$ and the reaction mixture was heated at $40{ }^{\circ} \mathrm{C}$. Sodium tungstate dihydrate $(5.09 \mathrm{~g}, 15.46 \mathrm{mmol})$ suspended in water $(1.99 \mathrm{~g})$ was added, followed by the urea-hydrogen peroxide complex $(72.71 \mathrm{~g}, 772.90 \mathrm{mmol})$ in two portions. The internal temperature of the flask was maintained below $50^{\circ} \mathrm{C}$ (water bath). The reaction was monitored by TLC (silica gel, ethyl acetate) and was completed after $3 \mathrm{~h}$. The reaction flask was cooled in an ice bath and $\mathrm{MnO}_{2}$ (Fluka, ref 63548, $10.0 \mathrm{~g}$ ) was added in three portions to destroy the excess of hydrogen peroxide. The reaction mixture was vigorously stirred until the end of dioxygen evolution (15 min) and the solvent was removed under vacuum. The residue was taken up in dichloromethane $(100 \mathrm{~mL})$, anhydrous $\mathrm{Na}_{2} \mathrm{SO}_{4}(40 \mathrm{~g})$ was added, and the reaction mixture was filtered over Celite. After concentration, the crude product was refluxed in ethyl acetate (32 $\mathrm{mL})$, the hot reaction mixture was filtered and cyclohexane $(29 \mathrm{~mL})$ was added portionwise to the boiling solution. The reaction mixture was allowed to cool slowly and after $4 \mathrm{~h}$ at room temperature, filtration yielded the nitrone $\mathbf{1}$ as white crystals (18.56 g, $109.04 \mathrm{mmol}, 54 \%$ from glycine ethyl ester hydrochloride); $\mathrm{mp} 113.0-113.1{ }^{\circ} \mathrm{C} ; R_{f}=0.32$ (ethyl acetate); $v_{\max }(\mathrm{KBr}$ pellet): 2972 , 2803, 1701, 1561, 1259 and 1046; $\delta_{\mathrm{H}}\left(400 \mathrm{MHz} ; \mathrm{CDCl}_{3} ; \mathrm{Me}_{4} \mathrm{Si}\right) 7.06(\mathrm{~s}, 1 \mathrm{H}), 2.99(\mathrm{~s}, 3 \mathrm{H}), 2.30(\mathrm{qq}, J 7.2$ and $6.8,1 \mathrm{H}), 1.66(\mathrm{~s}$, $3 \mathrm{H}), 1.00(\mathrm{~d}, J 7.2,3 \mathrm{H}), 0.97(\mathrm{~d}, J 6.8,3 \mathrm{H}) \mathrm{ppm} ; \delta_{\mathrm{C}}\left(100 \mathrm{MHz}, \mathrm{CDCl}_{3}\right)$ 162.8, 124.9, 94.1, 34.4, 26.0, 21.5, 16.1, 15.4 ppm; MS $\left(\mathrm{ES}^{+}\right): \mathrm{m} / z 363\left(2 \mathrm{M}+\mathrm{Na}^{+}, 23 \%\right), 341\left(2 \mathrm{M}+\mathrm{H}^{+}, 29\right), 193\left(\mathrm{M}+\mathrm{Na}^{+}, 57\right), 171\left(\mathrm{M}+\mathrm{H}^{+}, 100\right)$; Anal. Found: $\mathrm{C}, 56.08 ; \mathrm{H}, 8.26 ; \mathrm{N}$, 16.59; Calc. for $\mathrm{C}_{8} \mathrm{H}_{14} \mathrm{~N}_{2} \mathrm{O}_{2}$ : C, $56.45 ; \mathrm{H}, 8.29 ; \mathrm{N}, 16.46 \%$.

(rac)- and $(2 R, 3 \mathrm{a} R, 6 R)$ acetic acid (6-isopropyl-5,6-dimethyl-4-oxo-hexahydro-imidazo[1,5-b]isoxazol-2-yl)methyl ester 5a and (-)$5 a$ 
The preparation of $\mathbf{5 a}$ is typical: The reaction was performed in a sealed vessel. A mixture of nitrone $\mathbf{1}(340 \mathrm{mg}, 2.00 \mathrm{mmol})$ and allyl acetate $(2.16 \mathrm{~mL}, 20.00 \mathrm{mmol})$ was heated at $80{ }^{\circ} \mathrm{C}$ for $16 \mathrm{~h}$. The major part of the alkene was removed under reduced pressure. The residue was purified by column chromatography (silica gel, ethyl acetate/cyclohexane - 70:30) to yield isoxazolidine 5a (468 mg, $1.73 \mathrm{mmol}, 87 \%$ ) as a yellow oil; $R_{f}=0.35$ (ethyl acetate); $v_{\max }(\mathrm{KBr}$ pellet): 2973, 2935, 2890, 1735, 1696, 1407, 1382, 1260, 1235, 1084 and 1047; $\delta_{\mathrm{H}}\left(400 \mathrm{MHz} ; \mathrm{CDCl}_{3} ; \mathrm{Me}_{4} \mathrm{Si}\right) 4.16-3.98(\mathrm{~m}, 4 \mathrm{H}), 2.77(\mathrm{~s}, 3 \mathrm{H}), 2.65(\mathrm{ddd}, J 1.2,6.4$ and $12.4,1 \mathrm{H}), 2.24(\mathrm{td}, J 8.8$ and $12.4,1 \mathrm{H}), 2.05(\mathrm{~s}, 3 \mathrm{H}), 1.83(\mathrm{qq}, J 6.8$ and $7.2,1 \mathrm{H}), 1.45(\mathrm{~s}, 3 \mathrm{H}), 1.02(\mathrm{~d}, J 6.8,3 \mathrm{H}), 0.78(\mathrm{~d}, J$ 7.2, 3H) ppm; $\delta_{\mathrm{C}}\left(100 \mathrm{MHz}, \mathrm{CDCl}_{3}\right)$ 172.1, 170.7, 88.6, 74.5, 66.1, 64.1, 36.4, 35.2, 26.5, 20.9, 17.9, 17.0; MS $\left(\mathrm{ES}^{+}\right): \mathrm{m} / z .563$ $\left(2 \mathrm{M}+\mathrm{Na}^{+}, 11\right), 293\left(\mathrm{M}+\mathrm{Na}^{+}, 100\right), 271\left(\mathrm{M}+\mathrm{H}^{+}, 32\right)$; HRMS: $m / z$ 293.14756 $(\mathrm{M}+\mathrm{Na}) ; \mathrm{C}_{13} \mathrm{H}_{22} \mathrm{~N}_{2} \mathrm{O}_{4} \mathrm{Na}$ requires 293.14718.

(-)-5a (60 mg, $222 \mu \mathrm{mol}, 94 \%)$ was obtained starting from $(R)-\mathbf{1}(40 \mathrm{mg}, 235 \mu \mathrm{mol})$ and allyl acetate $(254 \mu \mathrm{L}, 2.35 \mathrm{mmol})$ as a pale yellow oil which solidified upon standing; $\mathrm{mp} 87.9-88.0{ }^{\circ} \mathrm{C} ;[\alpha]_{\mathrm{D}}{ }^{20}-37.9\left(c 1.06, \mathrm{CHCl}_{3}\right)$; Anal. Found: $\mathrm{C}, 57.47 ; \mathrm{H}, 8.43 ; \mathrm{N}$, 10.25; Calc. for $\mathrm{C}_{13} \mathrm{H}_{22} \mathrm{~N}_{2} \mathrm{O}_{4}$ : C, 57.76; H, 8.20; N, 10.36\%. The enantiomeric purity of (-)-5a ( $\geq 97 \%$ ee) was determined by chiral HPLC on a Daicel Chiralpak AD-RH column, 4.6 x $100 \mathrm{~mm}$, eluent water/acetonitrile - 60:40, 0.5 mL/min, retention time for (-)-5a $7.67 \mathrm{~min}$ and for (+)-5a $6.76 \mathrm{~min}$.

\section{(rac)- and $(2 R, 3 a R, 6 R)-2-H y d r o x y m e t h y l-6-i s o p r o p y l-5,6-d i m e t h y l-t e t r a h y d r o-i m i d a z o[1,5-b]$ isoxazol-4-one 5b and (-)-5b}

$\mathbf{5 b}$ was prepared as described for $\mathbf{5 a}$ using $\mathbf{1}(851 \mathrm{mg}, 5.00 \mathrm{mmol})$ and allyl alcohol $(3.4 \mathrm{~mL}, 50.00 \mathrm{mmol})$. The reaction mixture was heated at $80^{\circ} \mathrm{C}$ for $3.5 \mathrm{~h}$. Purification by column chromatography (silica gel, ethyl acetate/ethanol - 90:10) gave $\mathbf{5 b}(1.07 \mathrm{~g}$, $4.69 \mathrm{mmol}, 94 \%)$ as a pale yellow oil which solidified upon standing. An analytic sample crystallized from $t$-butyl methyl ether had a mp of 85.0-85.1 ${ }^{\circ} \mathrm{C} ; R_{f}=0.42$ (ethyl acetate/ethanol - 80:20); $v_{\max }(\mathrm{KBr}$ pellet): 3360, 2974, 2924, 2883, 1685, 1451, 1409, $1381,1089,1049$ and $881 ; \delta_{\mathrm{H}}\left(400 \mathrm{MHz} ; \mathrm{CDCl}_{3} ; \mathrm{Me}_{4} \mathrm{Si}\right) 4.07(\mathrm{br} \mathrm{d}, J$ 9.2, $1 \mathrm{H}), 4.04-4.00(\mathrm{~m}, 1 \mathrm{H}), 3.78$ (ddd, $J 2.8,5.2$ and 12.0 , $1 \mathrm{H}), 3.59(\mathrm{ddd}, J 2.0,6.8$ and $12.0,1 \mathrm{H}), 2.80(\mathrm{~s}, 3 \mathrm{H}), 2.63(\mathrm{ddd}, J 1.6,6.4$ and $12.4,1 \mathrm{H}), 2.39$ (ddd, $J 7.2,9.2$ and $12.4,1 \mathrm{H}), 2.20$ (br t, $J$ 6.0, $1 \mathrm{H}), 1.86$ (hept, $J 6.8,1 \mathrm{H}), 1.48(\mathrm{~s}, 3 \mathrm{H}), 1.04(\mathrm{~d}, J 6.8,3 \mathrm{H}), 0.83(\mathrm{~d}, J 6.8,3 \mathrm{H}) ; \delta_{\mathrm{C}}\left(100 \mathrm{MHz}, \mathrm{CDCl}_{3}\right) 172.4,88.4$, 77.3, 66.3, 63.7, 36.5, 33.9, 26.7, 17.8, 17.3, 17.1; $\mathrm{MS}\left(\mathrm{ES}^{+}\right): \mathrm{m} / z .779\left(2 \mathrm{M}+\mathrm{Na}^{+}, 42 \%\right), 251\left(\mathrm{M}+\mathrm{Na}^{+}, 100\right), 229\left(\mathrm{M}+\mathrm{H}^{+}, 54\right)$; HRMS: $m / z 251.13580(\mathrm{M}+\mathrm{Na}) ; \mathrm{C}_{11} \mathrm{H}_{20} \mathrm{~N}_{2} \mathrm{O}_{3} \mathrm{Na}$ : requires 251.13661 .

(-)-5b (46 mg, $201 \mu \mathrm{mol}, 86 \%)$ was obtained starting from $(R)-\mathbf{1}(40 \mathrm{mg}, 235 \mu \mathrm{mol})$ and allyl alcohol $(160 \mu \mathrm{L}, 2.35 \mathrm{mmol})$ as a pale yellow oil which solidified upon standing; $\mathrm{mp} 99.5-99.6{ }^{\circ} \mathrm{C} ;[\alpha]_{\mathrm{D}}{ }^{20}-32.7\left(c 1.08\right.$ in $\left.\mathrm{CHCl}_{3}\right)$; Anal. Found: $\mathrm{C}, 57.55$; $\mathrm{H}, 9.00$; N, 12.27; Calc. for $\mathrm{C}_{11} \mathrm{H}_{20} \mathrm{~N}_{2} \mathrm{O}_{3}:$ C, 57.87; H, 8.83; N, 12.27\%. The enantiomeric purity of (-)-5b ( $\geq 91 \%$ ee) was determined by chiral HPLC on a Daicel Chiralpak AD-RH column, 4.6 x $100 \mathrm{~mm}$, eluent water/acetonitrile $-60: 40,0.5 \mathrm{~mL} / \mathrm{min}$, retention time for (-)-5b $5.39 \mathrm{~min}$ and for (+)-5b $4.54 \mathrm{~min}$.

\section{(rac)-(2R*,3a $\left.R^{*}, 6 R^{*}\right)-6$-Isopropyl-5,6-dimethyl-2-phenyl-tetrahydro-imidazo[1,5-b]isoxazol-4-one $5 \mathrm{c}$}

5c was prepared as described for $\mathbf{5 a}$ using $\mathbf{1}(340 \mathrm{mg}, 2.00 \mathrm{mmol})$ and styrene $(2.3 \mathrm{~mL}, 20.00 \mathrm{mmol})$. The reaction mixture was heated at $80{ }^{\circ} \mathrm{C}$ for $3 \mathrm{~h}$. Purification by column chromatography (silica gel, ethyl acetate/cyclohexane - 70:30) gave 5c (494 mg, $1.80 \mathrm{mmol}, 90 \%$ ) as a colourless oil; $R_{f}=0.48$ (ethyl acetate); $v_{\max }(\mathrm{KBr}$ pellet): 3084, 3067, 3028, 2974, 2939, 2885, 1696, 1452, $1404,1082,1049,760,700 ; \delta_{\mathrm{H}}\left(400 \mathrm{MHz} ; \mathrm{CDCl}_{3} ; \mathrm{Me}_{4} \mathrm{Si}\right) 7.38-7.30(\mathrm{~m}, 5 \mathrm{H}), 4.73(\mathrm{dd}, J 5.0$ and 10.6, $1 \mathrm{H}), 4.20(\mathrm{br} \mathrm{d}, J 8.6,1 \mathrm{H})$, $2.95(\mathrm{dd}, J 5.0$ and $12.2,1 \mathrm{H}), 2.85(\mathrm{~s}, 3 \mathrm{H}), 2.52(\mathrm{ddd}, J 8.6,10.6$ and $12.2,1 \mathrm{H}), 1.92($ hept, $J 6.8,1 \mathrm{H}), 1.55(\mathrm{~s}, 3 \mathrm{H}), 1.09(\mathrm{~d}, J 6.8$, $3 \mathrm{H}), 0.83(\mathrm{~d}, J 6.8,3 \mathrm{H}) ; \delta_{\mathrm{C}}\left(100 \mathrm{MHz}, \mathrm{CDCl}_{3}\right)$ 172.1, 137.8, 128.7, 128.4, 126.7, 89.3, 79.1, 67.6, 41.4, 36.3, 26.4, 18.6, 17.0, 16.8; MS $\left(\mathrm{ES}^{+}\right): m / z 571\left(2 \mathrm{M}+\mathrm{Na}^{+}, 57 \%\right), 297\left(\mathrm{M}+\mathrm{Na}^{+}, 100\right), 275\left(\mathrm{M}+\mathrm{H}^{+}, 54\right) ; \mathrm{HRMS}: m / z 297.15803(\mathrm{M}+\mathrm{Na})$; $\mathrm{C}_{16} \mathrm{H}_{22} \mathrm{~N}_{2} \mathrm{O}_{2} \mathrm{Na}$ requires 297.15735.

\section{(rac)-(2R*,3a $\left.R^{*}, 6 R^{*}\right)-2-B u t o x y-6$-isopropyl-5,6-dimethyl-tetrahydro-imidazo[1,5-b]isoxazol-4-one 5d}

5d was prepared as described for 5a using $1(340 \mathrm{mg}, 2.00 \mathrm{mmol})$ and $n$-butyl vinyl ether $(2.6 \mathrm{~mL}, 20.00 \mathrm{mmol})$. The reaction mixture was heated at $80^{\circ} \mathrm{C}$ for $16 \mathrm{~h}$. Purification by column chromatography (silica gel, ethyl acetate/cyclohexane - 70:30) gave 5d (476 mg, $1.76 \mathrm{mmol}, 88 \%)$ as a colourless oil which solidified upon standing; $\mathrm{mp} 54.7-54.8{ }^{\circ} \mathrm{C} ; R_{f}=0.40$ (ethyl acetate/cyclohexane - 70:30); $v_{\max }\left(\mathrm{KBr}\right.$ pellet): 2958, 2921, 2873, 1691, 1458, 1432, 1401, 1386; $\delta_{\mathrm{H}}\left(400 \mathrm{MHz} \mathrm{CDCl}_{3}\right.$; $\left.\mathrm{Me}_{4} \mathrm{Si}\right) 5.01(\mathrm{dd}, J 1.4$ and $6.2,1 \mathrm{H}), 4.06(\mathrm{dd}, J 2.8$ and $9.6,1 \mathrm{H}), 3.68(\mathrm{td}, J 6.8$ and $10.0,1 \mathrm{H}), 3.40(\mathrm{td}, J 6.8$ and $10.0,1 \mathrm{H}), 2.80$ $(\mathrm{s}, 3 \mathrm{H}), 2.76(\mathrm{ddd}, J 2.8,6.2$ and $13.4,1 \mathrm{H}), 2.46(\mathrm{ddd}, J 1.4,9.6$ and $13.4,1 \mathrm{H}), 1.85$ (hept, $J 7.2,1 \mathrm{H}), 1.56($ quint, $J 6.8,2 \mathrm{H}), 1.43$ $(\mathrm{s}, 3 \mathrm{H}), 1.42-1.35(\mathrm{~m}, 2 \mathrm{H}), 1.03(\mathrm{~d}, J 7.2,3 \mathrm{H}), 0.91(\mathrm{~d}, J 7.2,3 \mathrm{H}), 0.91(\mathrm{t}, J 7.4,3 \mathrm{H}) ; \delta_{\mathrm{C}}\left(100 \mathrm{MHz}, \mathrm{CDCl}_{3}\right) 173.5,99.8,87.3$, 67.6, 64.1, 39.0, 37.0, 31.8, 27.3, 19.4, 18.1, 17.3, 17.0, 13.9; $\mathrm{MS}\left(\mathrm{ES}^{+}\right): \mathrm{m} / z .563\left(2 \mathrm{M}+\mathrm{Na}^{+}, 68 \%\right), 293(\mathrm{M}+\mathrm{Na}+100), 271(\mathrm{M}$ $+\mathrm{H}^{+}, 30$ ); Anal. Found: C, 62.36; H, 9.78; N, 10.40; Calc. for $\mathrm{C}_{14} \mathrm{H}_{26} \mathrm{~N}_{2} \mathrm{O}_{3}: \mathrm{C}, 62.20 ; \mathrm{H}, 6.70 ; \mathrm{N}, 10.37 \%$.

\section{$(r a c)-\left(2 R *, 3 a S^{*}, 6 R *\right)-6-I s o p r o p y l-5,6-d i m e t h y l-4-0 x o-h e x a h y d r o-i m i d a z o[1,5-b]$ isoxazole-2-carboxylic acid ethyl ester 5e}

5e was prepared as described for 5a using $\mathbf{1}(340 \mathrm{mg}, 2.00 \mathrm{mmol})$ and ethyl acrylate $(2.2 \mathrm{~mL}, 20.00 \mathrm{mmol})$. The reaction mixture was heated at $80{ }^{\circ} \mathrm{C}$ for $4 \mathrm{~h}$. Purification by column chromatography (silica gel, ethyl acetate/cyclohexane - 70:30) gave 5e (454 $\mathrm{mg}, 1.68 \mathrm{mmol}, 84 \%$ ) as a colourless oil; $R_{f}=0.42$ (ethyl acetate); $v_{\max }(\mathrm{KBr}$ pellet): 2973, 2918, 2883, 1740, 1686, 1459, 1399, $1378,1088,1049$ and $881 ; \delta_{\mathrm{H}}\left(400 \mathrm{MHz} ; \mathrm{CDCl}_{3} ; \mathrm{Me}_{4} \mathrm{Si}\right) 4.33(\mathrm{t}, J 6.8,1 \mathrm{H}), 4.24-4.10(\mathrm{~m}, 2 \mathrm{H}), 4.04(\mathrm{dd}, J 1.8$ and $9.2,1 \mathrm{H}), 2.83$ $(\mathrm{ddd}, J 1.8,7.2$ and $12.6,1 \mathrm{H}), 2.78(\mathrm{~s}, 3 \mathrm{H}), 2.74(\mathrm{ddd}, J 2.2,9.2$ and $12.6,1 \mathrm{H}), 1.82(\mathrm{qq}, J 6.8$ and $7.2,1 \mathrm{H}), 1.47(\mathrm{~s}, 3 \mathrm{H}), 1.26(\mathrm{t}$, $J 6.8,3 \mathrm{H}), 1.01(\mathrm{~d}, J 6.8,3 \mathrm{H}), 0.82(\mathrm{~d}, J 7.2,3 \mathrm{H}) ; \delta_{\mathrm{C}}\left(100 \mathrm{MHz}, \mathrm{CDCl}_{3}\right)$ 172.1, 170.4, 88.2, 74.3, 65.3, 61.5, 36.7, 35.8, 26.9, 17.6, 17.5, 17.1, 14.2; MS $\left(\mathrm{ES}^{+}\right): m / z 563\left(2 \mathrm{M}+\mathrm{Na}^{+}, 16 \%\right), 293\left(\mathrm{M}+\mathrm{Na}^{+}, 100\right), 271\left(\mathrm{M}+\mathrm{H}^{+}, 21\right) ; \mathrm{HRMS}: m / z 293.14783(\mathrm{M}+$ 
$\mathrm{Na}) ; \mathrm{C}_{13} \mathrm{H}_{22} \mathrm{~N}_{2} \mathrm{O}_{4} \mathrm{Na}$ requires 293.14718.

(rac)- and $(2 S, 3 R, 3 a R, 6 R)$-6-isopropyl-2,5,6-trimethyl-4-oxo-hexahydro-imidazo[1,5-b]isoxazole-3-carboxylic acid methyl ester 5f and (-)-5f

5f was prepared as described for $\mathbf{5 a}$ using $\mathbf{1}(340 \mathrm{mg}, 2.00 \mathrm{mmol})$ and trans-methyl crotonate $(2.1 \mathrm{~mL}, 20.00 \mathrm{mmol})$. The reaction mixture was heated at $80{ }^{\circ} \mathrm{C}$ for $16 \mathrm{~h}$. Purification by column chromatography (silica gel, ethyl acetate/cyclohexane - 70:30) gave 5f as a colourless oil (435 mg, $1.61 \mathrm{mmol}, 80 \%) ; R_{f}=0.41$ (ethyl acetate); $v_{\max }$ (KBr pellet): 2971, 2934, 2885, 1743, 1697, 1437, 1403, 1374, 1086 and 1050; $\delta_{\mathrm{H}}\left(400 \mathrm{MHz} ; \mathrm{CDCl}_{3} ; \mathrm{Me}_{4} \mathrm{Si}\right) 4.27(\mathrm{~d}, J$ 9.2, $1 \mathrm{H}), 4.08(\mathrm{qd}, J 6.0$ and 10.4, $1 \mathrm{H}), 3.78(\mathrm{~s}, 3 \mathrm{H}), 3.20(\mathrm{t}$, $J 9.2,1 \mathrm{H}), 2.75(\mathrm{~s}, 3 \mathrm{H}), 1.86(\mathrm{qq}, J 6.4$ and $6.8,1 \mathrm{H}), 1.49(\mathrm{~s}, 3 \mathrm{H}), 1.28(\mathrm{~d}, J 6.0,3 \mathrm{H}), 1.03(\mathrm{~d}, J 6.4,3 \mathrm{H}), 0.73(\mathrm{~d}, J 6.8,3 \mathrm{H}) ; \delta_{\mathrm{C}}$ $\left(100 \mathrm{MHz}, \mathrm{CDCl}_{3}\right)$ 169.2, 168.9, 89.1, 74.9, 68.0, 56.1, 52.2, 36.1, 26.2, 18.5, 16.9, 16.6, 16.5; MS $\left(\mathrm{ES}^{+}\right): \mathrm{m} / \mathrm{z} 563\left(2 \mathrm{M}+\mathrm{Na}^{+}\right.$, 26\%), $293\left(\mathrm{M}+\mathrm{Na}^{+}, 100\right), 271\left(\mathrm{M}+\mathrm{H}^{+}, 16\right)$; HRMS: $m / z 293.14770(\mathrm{M}+\mathrm{Na}) ; \mathrm{C}_{13} \mathrm{H}_{22} \mathrm{~N}_{2} \mathrm{O}_{4} \mathrm{Na}$ requires 293.14718.

(-)-5f $(84 \mathrm{mg}, 311 \mu \mathrm{mol}, 80 \%)$ was obtained starting from $(R)-\mathbf{1}(66 \mathrm{mg}, 388 \mu \mathrm{mol})$ and trans-methyl crotonate $(411 \mu \mathrm{L}, 3.88$ mmol) as a colourless oil; $[\alpha]_{\mathrm{D}}{ }^{20}-96.1\left(c 1.00, \mathrm{CHCl}_{3}\right)$.

The enantiomeric purity of (-)-5f ( $\geq 99 \%$ ee) was determined by chiral HPLC on a Daicel Chiralpak AD-RH column, $4.6 \times 100$ $\mathrm{mm}$, eluent water/acetonitrile $-60: 40,0.5 \mathrm{~mL} / \mathrm{min}$, retention time for (+)-5f $8.21 \mathrm{~min}$ and for (-)-5f $7.47 \mathrm{~min}$.

(rac)- and $(1 R, 3 \mathrm{a} R, 3 \mathrm{bS}, 7 \mathrm{a} S)$-1-Isopropyl-1,2-dimethyl-octahydro-8-oxa-2,8a-diaza-cyclopenta[a]inden-3-one $5 \mathrm{~g}$ and $(+)-5 \mathrm{~g}$

$\mathbf{5 g}$ was prepared as described for $\mathbf{5 a}$ using $1(340 \mathrm{mg}, 2.00 \mathrm{mmol})$, cyclohexene $(2.0 \mathrm{~mL}, 20.00 \mathrm{mmol})$ and toluene $(0.5 \mathrm{~mL})$. The reaction mixture was heated at $80{ }^{\circ} \mathrm{C}$ for $48 \mathrm{~h}(90 \%$ conversion). Purification by column chromatography (silica gel, ethyl acetate/cyclohexane - 65:35) gave $\mathbf{5 g}$ as a pale yellow oil (376 mg, $1.48 \mathrm{mmol}, 74 \%$ yield); $R_{f}=0.29$ (ethyl acetate/cyclohexane $70: 30) ; v_{\max }\left(\mathrm{KBr}\right.$ pellet): 2973, 2939, 2883, 1685, 1449, 1406, 1084 and 1050; $\delta_{\mathrm{H}}\left(400 \mathrm{MHz} ; \mathrm{CDCl}_{3} ; \mathrm{Me}_{4} \mathrm{Si}\right) 3.78-3.76(\mathrm{~m}, 1 \mathrm{H})$, $3.66(\mathrm{br} \mathrm{s}, 1 \mathrm{H}), 2.75(\mathrm{~s}, 3 \mathrm{H}), 2.63(\mathrm{ddd}, J 2.4,6.4$ and 12.0, $1 \mathrm{H}), 1.99-1.96(\mathrm{~m}, 1 \mathrm{H}), 1.83(\mathrm{qq}, J 6.4$ and $7.2,1 \mathrm{H}), 1.80-1.76(\mathrm{~m}$, $1 \mathrm{H}), 1.70-1.66(\mathrm{~m}, 1 \mathrm{H}), 1.55-1.41(\mathrm{~m}, 4 \mathrm{H}), 1.46(\mathrm{~s}, 3 \mathrm{H}), 1.24-1.13(\mathrm{~m}, 1 \mathrm{H}), 1.04(\mathrm{~d}, J 6.4,3 \mathrm{H}), 0.74(\mathrm{~d}, J 7.2,3 \mathrm{H}) ; \delta_{\mathrm{C}}(100 \mathrm{MHz}$, $\left.\mathrm{CDCl}_{3}\right)$ 172.1, 89.3, 74.8, 73.7, 45.1, 36.4, 27.3, 26.1, 25.5, 24.3, 19.8, 18.7, 17.1, 16.7; MS (ES $\left.{ }^{+}\right): m / z 527\left(2 \mathrm{M}+\mathrm{Na}^{+}, 26 \%\right)$, $275\left(\mathrm{M}+\mathrm{Na}^{+}, 42\right), 253\left(\mathrm{M}+\mathrm{H}^{+}, 100\right)$; HRMS: $m / z 275.17304(\mathrm{M}+\mathrm{Na}) ; \mathrm{C}_{14} \mathrm{H}_{24} \mathrm{~N}_{2} \mathrm{O}_{2} \mathrm{Na}$ requires 275.17300 .

$(+)-5 \mathrm{~g}(72 \mathrm{mg}, 285 \mu \mathrm{mol}, 65 \%$ yield) was obtained starting from $(R)-1$ (75 mg, $441 \mu \mathrm{mol})$ and cyclohexene $(447 \mu \mathrm{L}, 4.41$ $\mathrm{mmol})$ as a pale yellow oil; $[\alpha]_{\mathrm{D}}{ }^{20}+8.9\left(c 1.04\right.$ in $\left.\mathrm{CHCl}_{3}\right)$. The enantiomeric purity of $(+)-\mathbf{5 g}(\geq 99 \%$ ee $)$ was determined by chiral HPLC on a Daicel Chiralpak AD-RH column, 4.6 x $100 \mathrm{~mm}$, eluent water/acetonitrile - 68:32, 0.5 mL/min, retention time for (-)5g $19.30 \mathrm{~min}$ and for (+)-5g $17.70 \mathrm{~min}$.

( rac $)$-(1 $\left.R^{*}, 3 \mathrm{a} R^{*}, 3 \mathrm{~b} S^{*}, 4 S^{*}, 7 R^{*}, 7 \mathrm{a} S^{*}\right)-1-$ Isopropyl-1,2-dimethyl-octahydro-4,7-methano-8-oxa-2,8a-diaza-cyclopenta[a]inden-3one $5 \mathrm{~h}$

5h was prepared as described for $\mathbf{5 a}$ using $\mathbf{1}(170 \mathrm{mg}, 1.00 \mathrm{mmol})$, 2-norbornene (113 $\mathrm{mg}, 1.20 \mathrm{mmol})$ and toluene (1 mL). The reaction mixture was heated at $80{ }^{\circ} \mathrm{C}$ for $16 \mathrm{~h}$. Purification by column chromatography (silica gel, ethyl acetate/cyclohexane 65:35) gave $\mathbf{5 h}$ as a white solid (238 $\mathrm{mg}, 900 \mu \mathrm{mol}, 90 \%)$; $\mathrm{mp} 79.7-79.8{ }^{\circ} \mathrm{C} ; R_{f}=0.32$ (ethyl acetate/cyclohexane - 65:35); $v_{\max }$ (KBr pellet): 2958, 2873, 2360, 2339 and 1685; $\delta_{\mathrm{H}}\left(400 \mathrm{MHz} ; \mathrm{CDCl}_{3} ; \mathrm{Me}_{4} \mathrm{Si}\right) 3.99$ (br d, $J$ 6.8, $\left.1 \mathrm{H}\right), 3.61$ (d, $\left.J 2.4,1 \mathrm{H}\right), 2.76(\mathrm{~s}$, $3 \mathrm{H}), 2.72-2.70(\mathrm{~m}, 1 \mathrm{H}), 2.27-2.25(\mathrm{~m}, 2 \mathrm{H}), 1.87-1.85(\mathrm{~m}, 1 \mathrm{H}), 1.78($ hept, $J 6.8,1 \mathrm{H}), 1.47-1.42(\mathrm{~m}, 2 \mathrm{H}), 1.39(\mathrm{~s}, 3 \mathrm{H}), 1.18-1.13$ $(\mathrm{m}, 1 \mathrm{H}), 1.10-1.07(\mathrm{~m}, 1 \mathrm{H}), 1.01(\mathrm{~d}, J 6.8,3 \mathrm{H}), 0.99-0.94(\mathrm{~m}, 1 \mathrm{H}), 0.83(\mathrm{~d}, J 6.8,3 \mathrm{H}) ; \delta_{\mathrm{C}}\left(100 \mathrm{MHz}, \mathrm{CDCl}_{3}\right) \quad 171.8,87.8,86.0$, $70.8,55.8,41.9,41.0,36.6,33.0,27.5,26.7,23.5,17.8,17.5,17.1 ; \mathrm{MS}\left(\mathrm{ES}^{+}\right): \mathrm{m} / z$ 551 $\left(2 \mathrm{M}+\mathrm{Na}^{+}, 42\right), 287\left(\mathrm{M}+\mathrm{Na}^{+}, 20\right), 265$ $\left(\mathrm{M}+\mathrm{H}^{+}, 100\right)$; Anal. Found: $\mathrm{C}, 68.12 ; \mathrm{H}, 9.25 ; \mathrm{N}, 10.58$; Calc. for $\mathrm{C}_{15} \mathrm{H}_{24} \mathrm{~N}_{2} \mathrm{O}_{2}: \mathrm{C}, 68.15 ; \mathrm{H}, 9.15 ; \mathrm{N}, 10.60 \%$.

(rac)-(3a $\left.R^{*}, 6 R^{*}\right)$-6-isopropyl-5,6-dimethyl-2-phenyl-5,6-dihydro-3a $H$-imidazo[1,5-b]isoxazol-4-one 7

7 was prepared as described for $\mathbf{5 a}$ using $1(170 \mathrm{mg}, 1.00 \mathrm{mmol})$, phenylacetylene $(220 \mu \mathrm{L}, 2.00 \mathrm{mmol})$ and toluene $(1 \mathrm{~mL})$. The reaction mixture was heated at $80{ }^{\circ} \mathrm{C}$ for $16 \mathrm{~h}$. Purification by column chromatography (silica gel, ethyl acetate/cyclohexane $75: 25)$ gave 7 as a beige solid $(199 \mathrm{mg}, 730 \mu \mathrm{mol}, 73 \%)$; mp 146.8-146.9 ${ }^{\circ} \mathrm{C} ; R f=0.33$ (ethyl acetate); $v_{\max }(\mathrm{KBr}$ pellet): 3089 , 3067, 3037, 2963, 2937, 2898, 2872, 1702, 1451, 1422, 1391, 823 and 733; $\delta_{\mathrm{H}}\left(400 \mathrm{MHz} ; \mathrm{CDCl}_{3} ; \mathrm{Me}_{4} \mathrm{Si}\right) 8.04$ (dd, $J 1.2$ and 7.2, $2 \mathrm{H}), 7.61(\mathrm{tt}, J 1.2$ and $7.2,1 \mathrm{H}), 7.51(\mathrm{t}, J 7.2,2 \mathrm{H}), 3.52(\mathrm{~d}, J 5.2,1 \mathrm{H}), 3.18(\mathrm{~d}, J 5.2,1 \mathrm{H}), 2.64(\mathrm{~s}, 3 \mathrm{H}), 1.92(\mathrm{qq}, J 6.4$ and 6.8 , $1 \mathrm{H}), 1.16(\mathrm{~s}, 3 \mathrm{H}), 1.10(\mathrm{~d}, J 6.4,3 \mathrm{H}), 0.90\left(\mathrm{~d}, J\right.$ 6.8, 3H); $\delta_{\mathrm{C}}\left(100 \mathrm{MHz}, \mathrm{CDCl}_{3}\right)$ 194.2, 168.9, 137.8, 134.0, 129.0, 128.7, 84.1, $45.3,42.3,38.7,26.0,20.7,16.4,16.2 ; \mathrm{MS}\left(\mathrm{ES}^{+}\right): \mathrm{m} / z 567\left(2 \mathrm{M}+\mathrm{Na}^{+}, 52 \%\right), 545\left(2 \mathrm{M}+\mathrm{H}^{+}, 32\right), 295\left(\mathrm{M}+\mathrm{Na}{ }^{+}, 100\right), 273(\mathrm{M}+$ $\left.\mathrm{H}^{+}, 65\right)$; Anal. Found: C, 70.17; H, 7.58; N, 10.29; Calc. for $\mathrm{C}_{16} \mathrm{H}_{20} \mathrm{~N}_{2} \mathrm{O}_{2}$ : C, 70.57; H, 7.41; N, $10.29 \%$.

A monocyclic isomer 7' with two possible tautomeric structures was isolated in 20\% yield (yellow oil, $55 \mathrm{mg}, 202 \mu \mathrm{mol}$ ): $R f=$ 0.52 (ethyl acetate); $\delta_{\mathrm{H}}\left(400 \mathrm{MHz} ; \mathrm{CDCl}_{3} ; \mathrm{Me}_{4} \mathrm{Si}\right) 7.97(\mathrm{~d}, J 6.8,2 \mathrm{H}), 7.48-7.41(\mathrm{~m}, 3 \mathrm{H}), 6.51(\mathrm{~s}, 1 \mathrm{H}), 2.95$ (s, $\left.3 \mathrm{H}\right), 2.10(\mathrm{qq}, J$ 6.4 and $6.8,1 \mathrm{H}), 1.54(\mathrm{~s}, 3 \mathrm{H}), 1.10(\mathrm{~d}, J 6.8,3 \mathrm{H}), 0.67(\mathrm{~d}, J 6.4,3 \mathrm{H}) ; \delta_{\mathrm{C}}\left(100 \mathrm{MHz}, \mathrm{CDCl}_{3}\right) 191.2,162.6,150.4,138.9,131.8$, $128.5,127.5,86.8,80.6,34.7,25.0,23.9,17.1,15.4 ; \mathrm{MS}\left(\mathrm{ES}^{+}\right): m / z 567\left(2 \mathrm{M}+\mathrm{Na}^{+}, 100 \%\right), 295\left(\mathrm{M}+\mathrm{Na}^{+}, 27\right), 273\left(\mathrm{M}+\mathrm{H}^{+}, 16\right)$

(rac)-(2S*,5 $\left.R^{*}\right)-5$-[(2R*)-2-hydroxy-2-phenylethyl]-2-isopropyl-2,3-dimethylimidazolidin-4-one 8c

In a $25-\mathrm{mL}$ flask, zinc dust $(657 \mathrm{mg}, 10.06 \mathrm{mmol})$ was added in one portion to a solution of $\mathbf{5 c}(138 \mathrm{mg}, 0.503 \mathrm{mmol})$ in acetic acid $(1.7 \mathrm{~mL}$ ). The reaction was monitored by TLC (silica gel, ethyl acetate) and was completed within $3.5 \mathrm{~h}$ at room temperature. The reaction mixture was filtered and the solid was rinsed with dichloromethane. After concentration of the filtrate, the crude 
product was purified by column chromatography (silica gel, ethyl acetate/cyclohexane - 80:20) to yield 8c as a colourless oil (116 $\mathrm{mg}, 0.420 \mathrm{mmol}, 83 \%$ ) which solidified upon standing; $\mathrm{mp} \mathrm{84.8-84.9}{ }^{\circ} \mathrm{C} ; R f=0.32$ (ethyl acetate/cyclohexane $-80: 20$ ); $v_{\max }$ (KBr pellet): $3345,3066,3033,2970,2873,1677,1451,1405,764$ and 702; $\delta_{\mathrm{H}}\left(400 \mathrm{MHz} ; \mathrm{CDCl}_{3} ; \mathrm{Me}_{4} \mathrm{Si}\right) 7.41-7.39$ (m, 2H), 7.35-7.31 (m, 2H), 7.26-7.22 (m, 1H), $4.95(\mathrm{dd}, J 2.8$ and 8.8, $1 \mathrm{H}), 3.85(\mathrm{dd}, J 4.4$ and 7.2, $1 \mathrm{H}), 2.83(\mathrm{~s}, 3 \mathrm{H}), 2.10-1.94(\mathrm{~m}, 3 \mathrm{H})$, $1.42(\mathrm{~s}, 3 \mathrm{H}), 1.00(\mathrm{~d}, J 6.8,3 \mathrm{H}), 0.83(\mathrm{~d}, J 6.8,3 \mathrm{H}) ; \delta_{\mathrm{C}}\left(100 \mathrm{MHz}, \mathrm{CDCl}_{3}\right) 174.1,144.7,128.4,127.3,125.7,81.0,72.6,59.4$, 42.8, 35.3, 26.4, 25.7, 16.9, 16.6; MS $\left(\mathrm{ES}^{+}\right): \mathrm{m} / \mathrm{z} 575\left(2 \mathrm{M}+\mathrm{Na}^{+}, 12 \%\right), 277\left(\mathrm{M}+\mathrm{H}^{+}, 100\right)$; Anal. Found: $\mathrm{C}, 69.54 ; \mathrm{H}, 8.75 ; \mathrm{N}$, 10.25; Calc. for $\mathrm{C}_{16} \mathrm{H}_{24} \mathrm{~N}_{2} \mathrm{O}_{2}$ : C, $69.54 ; \mathrm{H}, 8.76 ; \mathrm{N}, 10.14 \%$.

(rac)-(2R*)-2-hydroxy-3-[(2S*,4R*)-1,2-dimethyl-5-oxo-2-(propan-2-yl)imidazolidin-4-yl]-propanoic acid ethyl ester 8e

In an argon-flushed 25-mL two-necked flask was introduced Pd/C $10 \%$ (Fluka, ref 75990, $67 \mathrm{mg}$ ), followed by $2 \mathrm{~mL}$ of methanol and then a solution of $\mathbf{5 c}(114 \mathrm{mg}, 422 \mu \mathrm{mol})$ in methanol $(2 \mathrm{~mL})$. Ammonium formate $(532 \mathrm{mg}, 8.43 \mathrm{mmol})$ was added in one portion and the argon inlet was removed. The reaction was monitored by TLC (silica gel, ethyl acetate) and was completed within $75 \mathrm{~min}$ at room temperature. The reaction mixture was filtered over Celite and the solid was rinsed with dichloromethane. The filtrate was diluted with water $(2.5 \mathrm{~mL})$, the aqueous layer was separated and extracted with dichloromethane $(5 \mathrm{~mL})$. The gathered organic layers were dried over anhydrous $\mathrm{Na}_{2} \mathrm{SO}_{4}$ and filtered. After concentration under reduced pressure, purification by column chromatography (silica gel, ethyl acetate/cyclohexane - 80:20) yielded amino alcohol 8e (84 mg, $308 \mu \mathrm{mol}, 73 \%)$ as a yellow oil; $R_{f}=0.26$ (ethyl acetate); $v_{\max }\left(\right.$ film): $3445,3348,2973,2937,2880,1739,1682,1473$ and $755 ; \delta_{\mathrm{H}}\left(400 \mathrm{MHz}^{2} \mathrm{CDCl}_{3}\right.$; $\left.\mathrm{Me}_{4} \mathrm{Si}\right) 4.39(\mathrm{t}, J 4.8,1 \mathrm{H}), 4.24(\mathrm{q}, J 7.2,2 \mathrm{H}), 3.78(\mathrm{t}, J 6.4,1 \mathrm{H}), 2.78(\mathrm{~s}, 3 \mathrm{H}), 2.10-2.07(\mathrm{~m}, 2 \mathrm{H}), 1.96($ hept $, J 6.8,1 \mathrm{H}), 1.40(\mathrm{~s}$, $3 \mathrm{H}), 1.29(\mathrm{t}, J 7.2,3 \mathrm{H}), 0.99(\mathrm{~d}, J 6.8,3 \mathrm{H}), 0.80(\mathrm{~d}, J 6.8,3 \mathrm{H}) ; \delta_{\mathrm{C}}\left(100 \mathrm{MHz}, \mathrm{CDCl}_{3}\right) 174.2,173.4,80.9,69.5,61.6,57.8,37.1$, 35.1, 26.4, 25.5, 16.8, 16.4, 14.3; MS $\left(\mathrm{ES}^{+}\right): m / z 567\left(2 \mathrm{M}+\mathrm{Na}^{+}, 5 \%\right), 295\left(\mathrm{M}+\mathrm{Na}^{+}, 29\right), 273\left(\mathrm{M}+\mathrm{H}^{+}, 100\right) ; \mathrm{HRMS}: m / z$ $295.16307(\mathrm{M}+\mathrm{Na}) ; \mathrm{C}_{13} \mathrm{H}_{24} \mathrm{~N}_{2} \mathrm{O}_{4} \mathrm{Na}$ requires 295.16338. (rac)- and (2S,3R)-3-Hydroxy-2-((2S,4R)-2-isopropyl-1,2-dimethyl-5-oxo-imidazolidin-4-yl)-butyric acid methyl ester 8f and (+)-
8f

8f was prepared as described for 8e using $5 \mathbf{f}(83 \mathrm{mg}, 307 \mu \mathrm{mol})$. Purification by column chromatography (silica gel, ethyl acetate/cyclohexane - 80:20 to 100:0) yielded amino alcohol $\mathbf{8 f}(57 \mathrm{mg}, 0.209 \mathrm{mmol}, 68 \%)$ as a colourless oil which solidified upon standing; $\mathrm{mp} 72.3-72.4{ }^{\circ} \mathrm{C} ; R_{f}=0.21$ (ethyl acetate/cyclohexane - 80:20); $v_{\max }$ (KBr pellet): 3373, 2974, 2927, 2883, 1719, $1677,1438,1407,1378,1088$ and $1050 ; \delta_{\mathrm{H}}\left(400 \mathrm{MHz} ; \mathrm{CDCl}_{3} ; \mathrm{Me}_{4} \mathrm{Si}\right) 4.24$ (quint, $J$ 6.4, $\left.1 \mathrm{H}\right), 4.04(\mathrm{~d}, J 6.4,1 \mathrm{H}), 3.70(\mathrm{~s}, 3 \mathrm{H})$, 2.82-2.76 (m, $1 \mathrm{H}), 2.80(\mathrm{~s}, 3 \mathrm{H}), 1.97($ hept $J 6.8,1 \mathrm{H}), 1.34(\mathrm{~s}, 3 \mathrm{H}), 1.23(\mathrm{~d}, J 6.4,3 \mathrm{H}), 0.96(\mathrm{~d}, J 6.8,3 \mathrm{H}), 0.81(\mathrm{~d}, J 6.8,3 \mathrm{H}) ; \delta_{\mathrm{C}}$ $\left(100 \mathrm{MHz}, \mathrm{CDCl}_{3}\right)$ 172.9, 172.7, 80.9, 67.2, 59.3, 56.2, 51.7, 35.2, 26.2, 25.5, 21.3, 16.8, 16.4; MS $\left(\mathrm{ES}^{+}\right): \mathrm{m} / \mathrm{z}, 567\left(2 \mathrm{M}+\mathrm{Na}^{+}\right.$, 5\%), $295\left(\mathrm{M}+\mathrm{Na}^{+}, 10\right), 273\left(\mathrm{M}+\mathrm{H}^{+}, 100\right)$; HRMS: $m / z$ 273.18081 $(\mathrm{M}+\mathrm{H}) ; \mathrm{C}_{13} \mathrm{H}_{25} \mathrm{~N}_{2} \mathrm{O}_{4}$ requires 273.18088 .

(+)-8f (49 mg, $180 \mu \mathrm{mol}, 67 \%)$ was obtained starting from (-)-5f (73 mg, $270 \mu \mathrm{mol})$ as a colourless oil which solidified on standing; mp 89.3-89.4 ${ }^{\circ} \mathrm{C} ;[\alpha]_{\mathrm{D}}{ }^{20}+47.1\left(c 1.06, \mathrm{CHCl}_{3}\right)$; Anal. Found: C, 57.59; H, 8.90; N, 10.12; Calc. for $\mathrm{C}_{13} \mathrm{H}_{24} \mathrm{~N}_{2} \mathrm{O}_{4}$ : $\mathrm{C}$, $57.33 ; \mathrm{H}, 8.88 ; \mathrm{N}, 10.29 \%$.

\section{(rac)-1-Hydroxy-2-isopropyl-2,3-dimethyl-imidazolidin-4-one 9}

In a $100-\mathrm{mL}$ flask under an argon atmosphere, nitrone 1 (3.40 g, $20.00 \mathrm{mmol})$ was dissolved in anhydrous THF (40 mL). Sodium borohydride $(1.51 \mathrm{~g}, 40.00 \mathrm{mmol})$ was added in one portion. The reaction mixture was stirred for $2.5 \mathrm{~h}$ at room temperature and was then treated with $\mathrm{H}_{2} \mathrm{O}(2.9 \mathrm{~mL}, 160.00 \mathrm{mmol})$. The reaction mixture was stirred until the end of dihydrogen evolution (15 $\mathrm{min})$ and then the solvent was removed under reduced pressure. The crude product was taken up in ethyl acetate $(50 \mathrm{~mL})$, the organic layer was separated from the aqueous one, dried over anhydrous $\mathrm{Na}_{2} \mathrm{SO}_{4}$ and filtered. The filtrate was concentrated under vacuum to yield pure $9\left(3.44 \mathrm{~g}, 20.00 \mathrm{mmol}\right.$, quantitative yield) as a pale yellow oil; $R_{f}=0.32$ (ethyl acetate); $v_{\max }($ film): 3352 , 2970, 2937, 2881, 2838, 1682, 1435 and 1405; $\delta_{\mathrm{H}}\left(400 \mathrm{MHz} ; \mathrm{CDCl}_{3} ; \mathrm{Me}_{4} \mathrm{Si}\right) 5.78(\mathrm{~s}, 1 \mathrm{H}), 3.79(\mathrm{~d}, J 16.0,1 \mathrm{H}), 3.57(\mathrm{~d}, J 16.0$, $1 \mathrm{H}), 2.77(\mathrm{~s}, 3 \mathrm{H}), 1.86($ hept, $J$ 6.8, $1 \mathrm{H}), 1.38(\mathrm{~s}, 3 \mathrm{H}), 1.01(\mathrm{~d}, J 6.8,3 \mathrm{H}), 0.84(\mathrm{~d}, J 6.8,3 \mathrm{H}) ; \delta_{\mathrm{C}}\left(100 \mathrm{MHz}, \mathrm{CDCl}_{3}\right) 170.1,88.3$, 59.8, 35.3, 26.1, 17.5, 17.2, 15.8; MS $\left(\mathrm{ES}^{+}\right): m / z 367\left(2 \mathrm{M}+\mathrm{Na}^{+}, 58 \%\right), 195\left(\mathrm{M}+\mathrm{Na}^{+}, 100\right), 173\left(\mathrm{M}+\mathrm{H}^{+}, 37\right) ; \mathrm{HRMS}: m / z$ 195.11024 $(\mathrm{M}+\mathrm{Na}) ; \mathrm{C}_{8} \mathrm{H}_{16} \mathrm{~N}_{2} \mathrm{O}_{2} \mathrm{Na}$ requires 195.11040.

\section{(S)-2-Methoxycarbonylamino-3-phenyl-propionic acid $(R)$-2-isopropyl-2,3-dimethyl-4-oxo-imidazolidin-1-yl-ester 10a}

Moc-Phe-OH (11.41 g, 51.11. mmol, 87\%) was prepared from L-phenylalanine (9.69 g, $58.66 \mathrm{mmol})$ according to ref. 23. In a $100-\mathrm{mL}$ flask, $N$-(methoxycarbonyl)-L-phenylalanine $(1.34 \mathrm{~g}, 6.00 \mathrm{mmol})$ was dissolved in dichloromethane $(10 \mathrm{~mL})$ and solid 1,3-dicyclohexylcarbodiimide $(1.24 \mathrm{~g}, 6.00 \mathrm{mmol})$ was added. A solution of hydroxylamine $9(861 \mathrm{mg}, 5.00 \mathrm{mmol}) \mathrm{in}$ dichloromethane $(10 \mathrm{~mL})$ was added rapidly at $0{ }^{\circ} \mathrm{C}$. The reaction mixture was stirred for $1 \mathrm{~h}$ at $0{ }^{\circ} \mathrm{C}$, then the urea was precipitated by addition of diethylether $(20 \mathrm{~mL})$ and filtered off. The filtrate was stirred for 5 minutes with a $1 \mathrm{M}$ aqueous solution of $\mathrm{HCl}(20 \mathrm{~mL})$; the aqueous phase was separated and extracted twice with diethylether $(50 \mathrm{~mL})$. The gathered organic layers were washed with a saturated aqueous solution of $\mathrm{NaHCO}_{3}(100 \mathrm{~mL})$, then with brine $(100 \mathrm{~mL})$, dried over anhydrous $\mathrm{Na}_{2} \mathrm{SO}_{4}$ and filtered. After removal of the solvents under reduced pressure, more urea was precipitated in diethylether and filtered off. Concentration of the filtrate under vacuum yielded the 1:1 mixture of diastereoisomers $\mathbf{1 0}$ as a white foam (1.55 g, 4.10 mmol). Separation by medium pressure liquid chromatography (silica gel, diethylether/dichloromethane - 10:90 to 100:0 in 15 min, 15 $\left.\mathrm{mL} \cdot \mathrm{min}^{-1}\right)$ yielded the diastereoisomer $10 \mathrm{a}(300 \mathrm{mg}, 0.795 \mathrm{mmol}, 32 \%)$ as a white solid; $\mathrm{mp} 121.3-121.4{ }^{\circ} \mathrm{C} ;[\alpha]_{\mathrm{D}}{ }^{20}+21.2(c 0.98$ 
in $\mathrm{CHCl}_{3}$ ); $R_{f}=0.38$ (dichloromethane/t-butyl methyl ether - 70:30); $v_{\max }(\mathrm{KBr}$ pellet): 3326, 3020, 2963, 2933, 2920, 1776, 1701, 1686, 1540, 1125, 751 and 710; $\delta_{\mathrm{H}}\left(400 \mathrm{MHz} ; \mathrm{CDCl}_{3} ; \mathrm{Me}_{4} \mathrm{Si}\right) 7.32-7.27(\mathrm{~m}, 3 \mathrm{H}), 7.17(\mathrm{~d}, J 6.4,2 \mathrm{H}), 5.22(\mathrm{br} \mathrm{d}, J 6.4,1 \mathrm{H}), 4.52-$ $4.47(\mathrm{~m}, 1 \mathrm{H}), 3.82(\mathrm{~d}, J 17.2,1 \mathrm{H}), 3.67(\mathrm{~s}, 3 \mathrm{H}), 3.07(\mathrm{dd}, J 6.8$ and 13.6, $1 \mathrm{H}), 3.01(\mathrm{~d}, J 17.2,1 \mathrm{H}), 2.98(\mathrm{dd}, J 8.0$ and $13.6,1 \mathrm{H})$, $2.79(\mathrm{~s}, 3 \mathrm{H}), 1.85$ (hept, $J$ 6.8, $1 \mathrm{H}), 1.30(\mathrm{~s}, 3 \mathrm{H}), 0.99(\mathrm{~d}, J 6.8,3 \mathrm{H}), 0.91(\mathrm{~d}, J 6.8,3 \mathrm{H}) ; \delta_{\mathrm{C}}\left(100 \mathrm{MHz} \mathrm{CDCl}_{3}\right) 170.4,169.5$, $156.3,135.3,129.4,129.0,127.6,89.5,58.7,54.1,52.6,38.7,36.1,26.6,17.5,17.2,16.4 ; \mathrm{MS}_{\left(\mathrm{ES}^{+}\right): m / z} 777\left(2 \mathrm{M}+\mathrm{Na}^{+}, 14 \%\right)$, $400\left(\mathrm{M}+\mathrm{Na}^{+}, 100\right), 378\left(\mathrm{M}+\mathrm{H}^{+}, 11\right), 177\left(\mathrm{M}+\mathrm{Na}-\mathrm{Moc}-\mathrm{Phe}-\mathrm{OH}^{+}, 39\right), 155\left(\mathrm{M}+\mathrm{H}-\right.$ Moc-Phe-OH $\left.{ }^{+}, 84\right)$; Anal. Found: C, $60.67 ; \mathrm{H}, 7.12 ; \mathrm{N}, 11.15$; Calc. for $\mathrm{C}_{19} \mathrm{H}_{27} \mathrm{~N}_{3} \mathrm{O}_{5}$ : C, 60.47; $\mathrm{H}, 7.21 ; \mathrm{N}, 11.14 \%$.

The relative configuration of $\mathbf{1 0 a}$ was determined by X-ray crystallography of an analytical sample recrystallized in ethanol.

\section{(R)-2-Isopropyl-2,3-dimethyl-1-oxy-2,3-dihydro-imidazol-4-one $(R)-1$}

In a 50-mL flask, 10a (394 mg, $1.04 \mathrm{mmol})$ was dissolved in methanol (15 mL). $\mathrm{NaHCO}_{3}(877 \mathrm{mg}, 10.44$ mmol) was added, followed by a $35 \%$ aqueous solution of hydrogen peroxide $(1.01 \mathrm{~g}, 10.44 \mathrm{mmol})$. The reaction was monitored by TLC (ethyl acetate); hydrolysis of the ester bond was completed within $4 \mathrm{~h}$ at room temperature. $\mathrm{MnO}_{2}$ (Fluka, ref 63548 , $600 \mathrm{mg}$ ) was then added portionwise, both to destroy the excess of hydrogen peroxide and complete the oxydation of the intermediate hydroxylamine. The reaction mixture was stirred for $15 \mathrm{~min}$ at room temperature and was then filtered over Celite. The solid was rinsed with ethyl acetate and the solvents were removed under vacuum. Purification by column chromatography (silica gel, ethyl acetate/cyclohexane - 70:30), followed by recrystallization from ethyl acetate/cyclohexane $(1.0 \mathrm{~mL} / 0.7 \mathrm{~mL})$ yielded the nitrone $(R)-\mathbf{1}$ as a beige solid (124 mg, $729.5 \mathrm{mmol}, 70 \%)$; $[\alpha]_{\mathrm{D}}{ }^{20}-28.5\left(c 3.96\right.$ in $\left.\mathrm{CHCl}_{3}\right)$. The enantiomeric purity of $(-)-(R)-\mathbf{1}(\geq 99 \%$ ee) was determined by chiral HPLC on a Daicel Chiralpak AD-RH column, 4.6 x $100 \mathrm{~mm}$, eluent acetonitrile/water - 70:30, 0.5 $\mathrm{mL} / \mathrm{min}$, retention time for $(R)-\mathbf{1} 6.96 \mathrm{~min}$ and for $(S)-15.94 \mathrm{~min}$.

\section{(rac)- and (3R,3aS,7aS)-(2-Oxo-octahydro-benzofuran-3-yl)-carbamic acid tert-butyl ester 11 and (-)-11}

In a $10-\mathrm{mL}$ microwave vial were introduced isoxazolidine $5 \mathbf{g}(85 \mathrm{mg}, 337 \mu \mathrm{mol})$, glacial acetic acid $(2 \mathrm{~mL})$, a $3 \mathrm{~N}$ aqueous solution of $\mathrm{HCl}(2 \mathrm{~mL})$ and $\mathrm{Pd} / \mathrm{C} 10 \%$ (Fluka, ref 15990, $20 \mathrm{mg}$ ). The vial was brought into the microwave reactor, evacuated, filled up with $\mathrm{H}_{2}$ and the reaction mixture was stirred under $\mathrm{H}_{2}$ pressure $(7 \mathrm{bar})$ for 15 min at $150{ }^{\circ} \mathrm{C}$. After filtration through Celite, the solids were rinsed with acetic acid and concentration under vacuum yielded the acetate salt of the lactone.

The crude salt was dissolved in methanol $(3 \mathrm{~mL})$ and added to a mixture of triethylamine $(0.12 \mathrm{~mL}, 842 \mu \mathrm{mol})$ and methanol $(1$ $\mathrm{mL})$, followed by di-t-butyldicarbonate $(147 \mathrm{mg}, 674 \mu \mathrm{mol})$. The reaction mixture was stirred for $4.5 \mathrm{~h}$ at $45{ }^{\circ} \mathrm{C}$, then concentrated under reduced pressure. Purification by column chromatography (silica gel, dichloromethane/t-butyl methyl ether 98:2) yielded $N$-Boc lactone 11 ( $35 \mathrm{mg}, 137 \mu \mathrm{mol}, 42 \%)$ as a colourless oil which solidified upon standing; $\mathrm{mp} 95.6-95.7^{\circ} \mathrm{C} ; R_{f}=$ 0.26 (dichloromethane/t-butyl methyl ether - 97:3); $v_{\max }$ (KBr pellet): 3328, 2994, 2975, 2937, 2663, 1787, 1684, 1527, 1164 and $967 ; \delta_{\mathrm{H}}\left(400 \mathrm{MHz} ; \mathrm{CDCl}_{3} ; \mathrm{Me}_{4} \mathrm{Si}\right) 5.00(\mathrm{br} \mathrm{s}, 1 \mathrm{H}), 4.54-4.50(\mathrm{~m}, 2 \mathrm{H}), 2.73-2.68(\mathrm{~m}, 1 \mathrm{H}), 2.26-2.23(\mathrm{~m}, 1 \mathrm{H}), 1.76-1.54(\mathrm{~m}, 4 \mathrm{H})$, $1.45(\mathrm{~s}, 9 \mathrm{H}), 1.41-1.29(\mathrm{~m}, 1 \mathrm{H}), 1.19(\mathrm{qt}, J 2.8$ and $13.2,1 \mathrm{H}), 0.91(\mathrm{dq}, J 3.2$ and $13.2,1 \mathrm{H}) ; \delta_{\mathrm{C}}\left(100 \mathrm{MHz}, \mathrm{CDCl}_{3}\right) 175.8,155.6$, 80.4, 76.8, 57.3, 39.4, 28.4, 27.4, 22.8, 22.0, 19.7; $\mathrm{MS}\left(\mathrm{ES}^{+}\right): \mathrm{m} / z 533\left(2 \mathrm{M}+\mathrm{Na}^{+}, 47 \%\right), 278\left(\mathrm{M}+\mathrm{Na}^{+}, 100\right)$; Anal. Found: C, $60.94 ; \mathrm{H}, 8.47 ; \mathrm{N}, 5.21$; Calc. for $\mathrm{C}_{13} \mathrm{H}_{21} \mathrm{NO}_{4}$ : C, 61.16; H, 8.29; N, 5.49\%.

(-)-11 (26 mg, $102 \mu \mathrm{mol}, 42 \%)$ was obtained starting from (+)-5g (62 $\mathrm{mg}, 243 \mu \mathrm{mol})$ as a pale yellow oil which solidified upon standing; mp $112.4-112.6{ }^{\circ} \mathrm{C} ;[\alpha]_{\mathrm{D}}{ }^{20}-78.1\left(c 1.04\right.$ in $\left.\mathrm{CHCl}_{3}\right)$.

\section{Acknowledgment}

The authors thank Beatrice Gennaro and Corinne Bailly for their assistance in NMR and X-ray analysis respectively, and Alicia Contet and Olga N. Burchak for their support. We are grateful to Université Joseph Fourier and the CNRS for financial support.

\section{Notes and references}

Département de Chimie Moléculaire, UMR-5250, ICMG FR-2607, CNRS, Université Joseph Fourier, BP-53, 38041 Grenoble Cedex 9, France; Veronique.Blandin@ujf-grenoble.fr

$\dagger$ Electronic Supplementary Information (ESI) available: copies of ${ }^{1} \mathrm{H}$ and ${ }^{13} \mathrm{C}$ NMR spectra for all new compounds, NOESY spectra for $\mathbf{5 f}$ and $\mathbf{5 h}$, description of the microwave irradiation parameters, ORTEP drawings of 1 and 10a. See DOI: 10.1039/b000000x/

1 See for instance: (a) I. A. Grigor'ev, in Nitrile Oxides, Nitrones, and Nitronates in Organic Synthesis. 2nd ed.; H. Feuer Ed.; Wiley-Interscience, 2008; pp. 129-434; (b) a recent review on asymmetric 1,3-dipolar cycloadditions: H. Pelissier, Tetrahedron, 2007, 63, 3235-3285.

2 Recent examples: (a) L. Manzoni, D. Arosio, L. Belvisi, A. Bracci, M. Colombo, D. Invernizzi and C. Scolastico, J. Org. Chem., 2005, 70, 41244132; (b) O. Tamura, T. Shiro, M. Ogasawara, A. Toyao and H. Ishibashi, J. Org. Chem., 2005, 70, 4569-4577; (c) G. Romeo, D. Iannazzo, A. Piperno, R. Romeo, A. Corsaro, A. Rescifina and U. Chiacchio, Mini-Rev. Org. Chem., 2005, 2, 59-77; (d) F. M. Cordero, S. Bonollo, F. Machetti, and A. Brandi, Eur. J. Org. Chem., 2006, 3235-3241; (e) K. Aouadi, E. Jeanneau, M. Msaddek and J.-P. Praly, Tetrahedron: Asymmetry 2008, 19, 1145-1152; $(f)$ T. B. Nguyen,; T. M. H. Vuong, A. Martel, R. Dhal and G. Dujardin, Tetrahedron: Asymmetry, 2008, 19, $2084-2087$.

3 See in particular the discussion in: O. Tamura, N. Mita, Y. Imai, T. Nishimura, T. Kiyotani, M. Yamasaki, M. Shiro, N. Morita, I. Okamoto, T. Takeya, H. Ishibashi and M. Sakamoto, Tetrahedron, 2006, 62, 12227-12236.

4 (a) N. Katagiri, M. Okada, C. Kaneko, and T. Furuya, Tetrahedron Lett., 1996, 37, 1801-1804; (b) B. Westermann, A. Walter, U. Florke and H.-J. Altenbach, Org. Lett., 2001, 3, 1375-1378; (c) S. W. Baldwin and A. Long, Org. Lett., 2004, 6, 1653-1656; (d) F. Cantagrel, S. Pinet, Y. Gimbert and P. Y. Chavant, Eur. J. Org. Chem., 2005, 2694-2701; (e) A. Pernet-Poil-Chevrier, F. Cantagrel, K. Le Jeune, C. Philouze and P. Y. Chavant, Tetrahedron: Asymmetry, 2006, 17, 1969-1974. 
5 (a) O. Tamura, K. Gotanda, R. Terashima, M. Kikuchi, T. Miyawaki and M. Sakamoto, Chem. Commun., 1996, 1861-1862; (b) S. W. Baldwin, B. G. Young and A. T. McPhail, Tetrahedron Lett., 1998, 39, 6819-6822; (c) F. Heaney, J. Fenlon, O. M. C, P. McArdle and D. Cunningham, J. Chem. Soc., Perkin Trans. 1, 2001, 3382-3392; (d) P.-F.Wang, P. Gao and P.-F. Xu, Synlett, 2006, 1095-1099.

6 For other enantiopure cyclic nitrones see the review: J. Revuelta, S. Cicchi, A. Goti and A. Brandi, Synthesis, $2007,485-504$.

7 (a) P. Maire, V. Blandin, M. Lopez and Y. Vallée, Synlett, 2003, 671-674; (b) J. Lawrence, L. Cointeaux, P. Maire, Y. Vallée and V. Blandin, Org. Biomol. Chem., 2006, 3125-3141; (c) J. Lawrence, M. Jourdan, Y. Vallée and V. Blandin, Org. Biomol. Chem., 2008, 6, 4575-4581.

8 (a) S. U. Pandya, C. Garcon, P. Y. Chavant, S. Py and Y. Vallée, Chem. Commun., 2001, 1806-1807; (b) S. Pinet, S. U. Pandya, P. Y. Chavant, A. Ayling and Y. Vallée, Org. Lett., 2002, 4, 1463-1466; (c) S. U. Pandya, S. Pinet, P. Y. Chavant and Y. Vallée, Eur. J. Org. Chem., $2003,3621-3627$.

9 See for instance the review: D. Seebach, A. R. Sting, M. Hoffmann, Angew. Chem. Int. Ed. Engl., 1996, 35, $2708-2748$.

10 (a) N. PraveenGanesh, S. d'Hondt and P. Y. Chavant, J. Org. Chem., 2007, 72, 4510-4514; (b) N. PraveenGanesh and P. Y. Chavant, Eur. J. Org. Chem., 2008, 4690-4696.

11 E. Marcantoni, M. Petrini and O. Polimanti, Tetrahedron Lett., 1995, 36, 3561-3562.

12 Crystal data for $1\left(\mathrm{C}_{8} \mathrm{H}_{14} \mathrm{~N}_{2} \mathrm{O}_{2}\right.$, racemic sample $) . M$ : 170.21 g.mol ${ }^{-1}$; crystal system: triclinic; unit-cell dimensions and volume: a $=6.900(2) \AA$, $\mathrm{b}=$ 7.365(2) $\AA, c=11.610(3) \AA, \alpha=83.78(2)^{\circ}, \beta=86.06(2)^{\circ}, \gamma=84.78(2)^{\circ}, \mathrm{V}=442.9(5) \AA^{3}$, temperature of measurement: $293.0 \mathrm{~K}$; space group symbol: P -1; No. of formula units in the unit cell $Z=2$; No. of measured and independent reflections: 9127 and $2392, R_{\text {int }}: 0.09$; refinement vs. F; final $R$ values (observed data): $R=0.0533, w R=0.0867$. For the ORTEP drawing of 1, see Electronic Supporting Information.

13 The reaction between MiPNO and cyclohexene, sluggish in toluene, was also performed in a polar solvent, NMP, without any improvement: 48 h, 64 $\%$ conversion.

14 The stereochemistry of the cycloadducts was assigned on the basis of their NOESY spectra.

15 Such a ring opening was previously reported: A. Padwa, M. Meske and Z. Ni, Tetrahedron Lett., 1993, 34, 5047-5050.

16 Attempted NOESY experiments were not conclusive enough to allow determination of the relative configuration of the minor isomer.

17 O. N. Burchak, C. Philouze, P. Y. Chavant and S. Py, Org. Lett., 2008, 10, 3021-3023.

18 Moc-Phe-OH ( $N$-methoxycarbonyl-L-phenylalanine): $(S)$-2-methoxycarbonylamino-3-phenylpropanoic acid

19 Crystal data for 10a $\left(\mathrm{C}_{19} \mathrm{H}_{27} \mathrm{~N}_{3} \mathrm{O}_{5}\right.$, enantiopure sample). $M$ : 377.44 g.mol ${ }^{-1}$; crystal system: monoclinic; unit-cell dimensions and volume: a = 10.080(3) $\AA, b=9.129(4) \AA, c=11.610(3) \AA, \beta=112.49(3)^{\circ}, \mathrm{V}=987.2(6) \AA^{3}$, temperature of measurement: $293.0 \mathrm{~K}$; space group symbol: P $2_{1}$; No. of formula units in the unit cell $Z=2$; No. of measured and independent reflections: 2273 and $2174, R_{\text {int }}$ : 0.07 ; refinement vs. F; final $R$ values (observed data): $R=0.0531, w R=0.0921$. The relative streochemistry $(R)$ at C5 was attributed knowing the $(S)$ configuration at $\mathrm{C} 11$ from the starting material Moc-Phe-OH.

20 Note that this step produces only volatile side products (3-methyl-butanone and methylamine).

21 For related compounds, see: (a) B. Merla, H.-J. Grumbach and N. Risch, Synthesis, 1998, 1609-1614; (b) W. Notz, S.-i. Watanabe, N. S. Chowdari, G. Zhong, J. M. Betancort, F. Tanaka and C. F. Barbas III, Adv. Synth. Catal., 2004, 346, 1131-1140.

22 R. Fitzi and D. Seebach, Angew. Chem. Int. Ed. Engl., 1986, 25, 345-346.

23 M. W. Liladhar, J. J. McKenna, A. Bach, M. Prashad, O. Repic and T. J. Blacklock, Synth. Comm., 2007, $37,1445-1454$. 